\title{
PROTECTIVE EFFECTS OF ISOLIQUIRITIN ON BEAS-2B CELLS AGAINST LPS-INDUCED OXIDATIVE STRESS AND INFLAMMATION INVOLVING ACTIVATION OF NRF2
}

\author{
LIANG-SHU CHEN ${ }^{1}$ and DONG-SHU ZHENG ${ }^{2 *}$ \\ ${ }^{1}$ Ward of Cadre Care, The First Affiliated Hospital of Xiamen University, \\ Xiamen, Fujian 361003, P.R. China \\ ${ }^{2}$ Department of Otolaryngology, Head and Neck Surgery, \\ The First Affiliated Hospital of Xiamen University, \\ Xiamen, Fujian 361003, P.R. China
}

\begin{abstract}
Inflammatory respiratory diseases such as chronic obstructive pulmonary disease (COPD) threaten people's health worldwide. To discover novel drugs targeting these diseases, we have evaluated the protective effects of isoliquiritin using LPS-treated BEAS-2B cells. As a result, isoliquiritin at $5 \mu \mathrm{M}$ can attenuate oxidative stress by reducing the ROS level and MDA content as well as elevating the activity of SOD and CAT. Meanwhile, the inflammation induced by LPS in BEAS-2B cells was ameliorated through decreasing the levels of TNF- $\alpha$, IL-1 $\beta$, and IL- 6 as well as inactivating the $\mathrm{NF}-\kappa \mathrm{B}$ pathway. In addition, activation of Nrf2 was found in LPS-induced BEAS-2B cells, which indicated the protective effects of isoliquiritin were associated with activation of Nrf2. These findings can give evidence for the application of isoliquiritin and the discovery of novel therapy for inflammatory pulmonary disorders.
\end{abstract}

Keywords: isoliquiritin, BEAS-2B cells, oxidative stress, inflammation, Nrf2

Pulmonary inflammation is considered as the body's response to the injury, which is involved in many respiratory diseases such as acute respiratory distress syndrome (ARDS), chronic obstructive pulmonary disease (COPD), and so on (1). Of these respiratory diseases, COPD is the major one resulting in death all over the world, which has been the global burden due to the increasing cigarette smoking and exacerbation of air pollution (2). In the pathogenesis of pulmonary diseases like COPD and ARDS, inflammation together with oxidative stress plays a pivotal role $(3,4)$. Therefore, targeting inflammation and oxidative stress can attenuate these pulmonary diseases. Nuclear factor erythroid 2-related factor 2 (Nrf2) is a transcription factor regulating the expression of enzymes for cell defense (5). Activation of Nrf2 can attenuate inflammatory diseases (6).

In searching for drugs for the treatment of pulmonary diseases related to inflammation, phytochemicals play a pivotal role such as curcumin (7), amygdalin (8), and so on. In addition, flavonoids including chalcones usually showed anti-inflammatory effects in many diseases (9). As a chalcone, isoliquiritin (Figure 1) is a bioactive phytochemical found in licorice (710). Previous pharmacological investigations revealed it possessed various activities including anti-inflammation (11), neuroprotection (12), anti-fungus (13), anti-depression (14), renoprotection (15), and so on. In our interest in the discovery of bioactive phytochemicals to alleviate pulmonary inflammatory diseases, we have investigated isoliquiritin using human lung bronchial epithelial BEAS-2B cells.

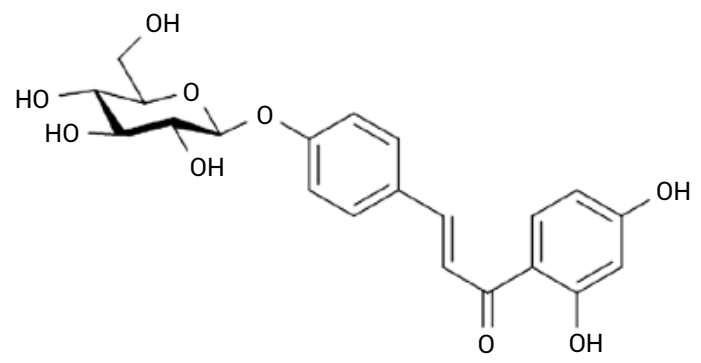

Figure 1. Chemical structure of isoliquiritin.

* Corresponding author: e-mail: noordaoud@hu.edu.jo,nor8150592@fgs.ju.edu.jo 


\section{MATERIALS AND METHODS}

\section{Chemicals and reagents}

Isoliquiritin (ILN) and sulforaphane (SFN), an Nrf2 activator, were purchased from Yuanye Biotechnology (Shanghai, China). All antibodies were provided by Abcam (Cambridge, UK). The assay kits used herein were supplied by Beyotime (Shanghai, China).

\section{Cell culture and treatment}

Human lung bronchial epithelial BEAS-2B cells were obtained from American Type Culture Collection (ATCC) and maintained in DMEM under an atmosphere of $5 \% \mathrm{CO}_{2}$ at $37^{\circ} \mathrm{C}$. Cells were divided into the control group, lipopolysaccharide (LPS) group, and experimental groups. The cells in the LPS group were treated with $1 \mu \mathrm{g} / \mathrm{mL}$ LPS in DMEM for $24 \mathrm{~h}$. The cells in experimental groups were incubated with $1 \mu \mathrm{g} / \mathrm{mL}$ LPS together with certain isoliquiritin or SFN for $24 \mathrm{~h}$. The cells of the control group were cultured in normal DMEM.

\section{Cell viability assay}

To determine the cell viability, an MTT assay was performed. In brief, the cells were seeded in 96-well microplates and treated as above. Then the cells were incubated with $0.5 \mathrm{mg} / \mathrm{mL}$ MTT for $4 \mathrm{~h}$ at $37^{\circ} \mathrm{C}$. Subsequently, the absorbance was read on a microplate reader (Molecular Devices, CA) at $570 \mathrm{~nm}$.

\section{ROS generation}

The level of ROS in BEAS-2B cells was detected using DCFH-DA in the assay kit. Briefly, the treated cells were exposed to $0.5 \mathrm{mg} / \mathrm{mL}$ DCFH-DA for $20 \mathrm{~min}$, and then the fluorescence intensity was recorded on a microplate reader at $488 \mathrm{~nm}$ for excitation wavelength and $525 \mathrm{~nm}$ for emission wavelength.

\section{MDA content}

A commercially available assay kit for the thiobarbituric acid method was used to determine the content of MDA. According to the supplier's protocol, the cells were treated as above and the supernatant was collected as a sample. After adding a working solution to the assay kit, the sample was boiled for $15 \mathrm{~min}$ and then cooled to room temperature. Next, the absorbance was measured at $532 \mathrm{~nm}$ using a microplate reader.

\section{SOD and CAT activity}

The activity of SOD and CAT was determined by the colorimetric method using the assay kits. The treated cells were lysed on ice using lysis solution and the supernatant was sucked for analysis after determination of the protein concentration using a BCA assay kit. The enzyme activity was measured in light of the supplier's instructions and the absorbance was monitored on a microplate reader at $520 \mathrm{~nm}$.

\section{ELISA assay}

To disclose the secretion of pro-inflammatory cytokines including TNF- $\alpha$, IL- $1 \beta$, and IL- 6 , commercially available ELISA was employed. In short, after treatment as above, the cells were centrifuged and the supernatant was collected as a sample. Then the sample was treated according to the supplier's instructions and the absorbance was recorded on a microplate reader at $450 \mathrm{~nm}$.

\section{Western blot analysis}

The total and nuclear proteins were extracted using RIPA solution and nuclear and cytoplasmic protein extraction kit. After quantification of the protein concentration using the BCA assay kit, the proteins were separated on 10\% SDS-PAGE and transferred to PVDF membranes. After blocking with defatted milk, the membranes were exposed to primary antibodies including $\mathrm{p}-\mathrm{I} \kappa \mathrm{B} \alpha$ (1 : 1000), p-NF-кB (1: 1000), Nrf2 (1: 1000), $\beta$-actin $(1: 1000)$, and lamin B1 (1: 1000) overnight. Then the membranes were rinsed with a buffer solution containing $0.1 \%$ Tween-20 and incubated with horseradish peroxidase-conjugated secondary antibody ( $1: 2000)$ for $2 \mathrm{~h}$. The bands were visualized with ECL substrate and analyzed using Image $\mathrm{J}$ software (NIH, Bethesda, MD). Since $\beta$-actin mainly exists in the cytosol, lamin B1 was employed as an internal control to analyze the nuclear Nrf2 in western blot.

\section{Immunofluorescence staining}

To localize intracellular Nrf2 in BEAS-2B cells, immunofluorescence staining was implemented. The cells were transferred into 12 -well microplates with a coverslip in each well and treated as above. Then, 4\% paraformaldehyde was employed to fix the cells and PBS containing $0.1 \%$ Triton $\mathrm{X}-100$ was used to increase the permeability of the cells. After blocking with 1\% BSA, the cells were subjected to the antibody of Nrf2 (1:200). After incubation overnight, the cells were exposed to DyLight 594-conjugated secondary antibody at $37^{\circ} \mathrm{C}$ in the dark. Then the cells were washed with PBS and treated with DAPI in the dark. Finally, the images were obtained using a fluorescence microscope (Nikon, Tokyo, Japan). 
Figure 2. Effects of isoliquiritin (ILN) on the survival of BEAS-2B cells.

\section{Statistical analysis}

All the experimental data were presented as mean \pm standard error and analyzed by GraphPad Prism 5.0 (GraphPad Software, La Jolla, CA). One-way ANOVA followed by Tukey test was implemented for multiple comparisons and Student's t-test single comparisons. $\mathrm{P}<0.05$ was considered as a significant difference in statistics.

\section{RESULTS}

\section{Effects of isoliquiritin on the viability of BEAS-2B cells}

As shown in Figure 2, in the presence of certain isoliquiritin from 0.01 to $200 \mu \mathrm{M}$, there is no significant difference compared to the control group. These results indicated isoliquiritin has no cytotoxic effects on normal BEAS-2B cells when the concentration is lower than $200 \mu \mathrm{M}$.

(A)

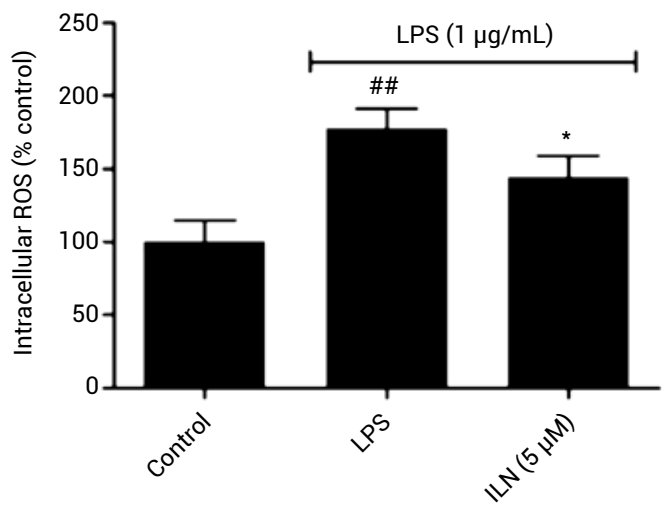

(C)

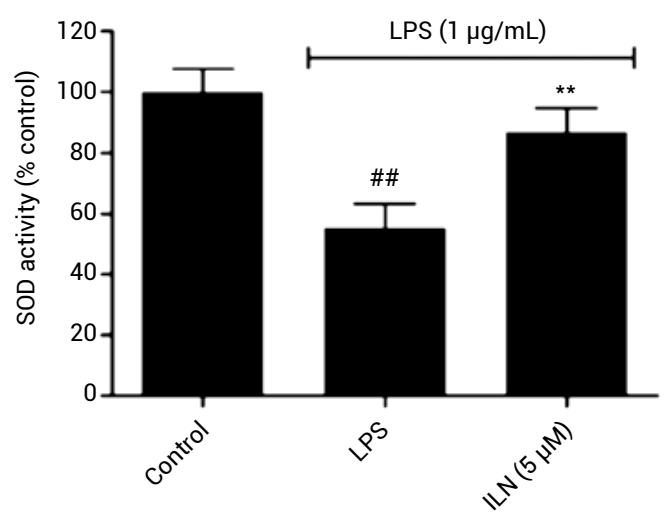

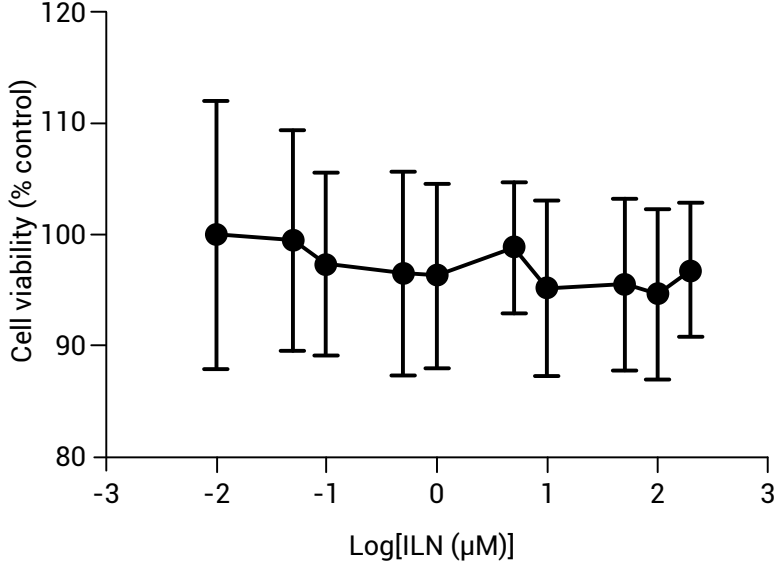

Effects of isoliquiritin on the oxidative stress induced by LPS in BEAS-2B cells

As the major initiator of oxidative stress, intracellular ROS levels in BEAS-2B cells were detected. The results showed LPS could induce the increasing ROS in BEAS-2B cells $(177.6 \pm 13.7 \%$ ), while isoliquiritin can reduce the level of excessive ROS (143.9 $\pm 14.8 \%$ ) (Figure 3A). At the same time,

(B)

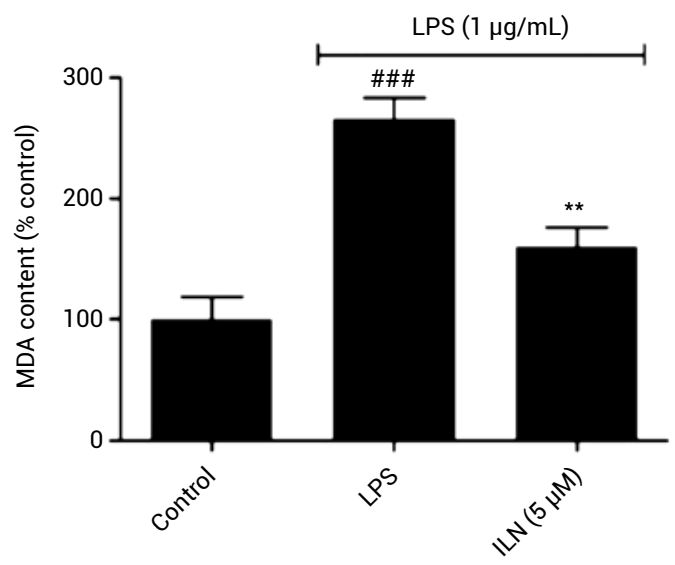

(D)

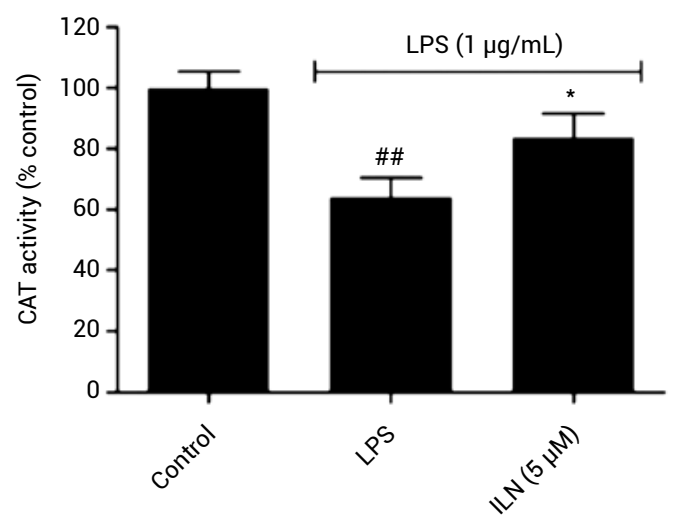

Figure 3. Effects of isoliquiritin (ILN) on the oxidative stress induce by LPS in BEAS-2B cells. (A) Intracellualr ROS level. (B) MDA content. (C) SOD activity. (D) CAT activity. $\mathrm{n}=3$, $\# \mathrm{P}<0.05$ vs control group, $* \mathrm{P}<0.05$, and $* * \mathrm{P}<0.01$ vs LPS group. 

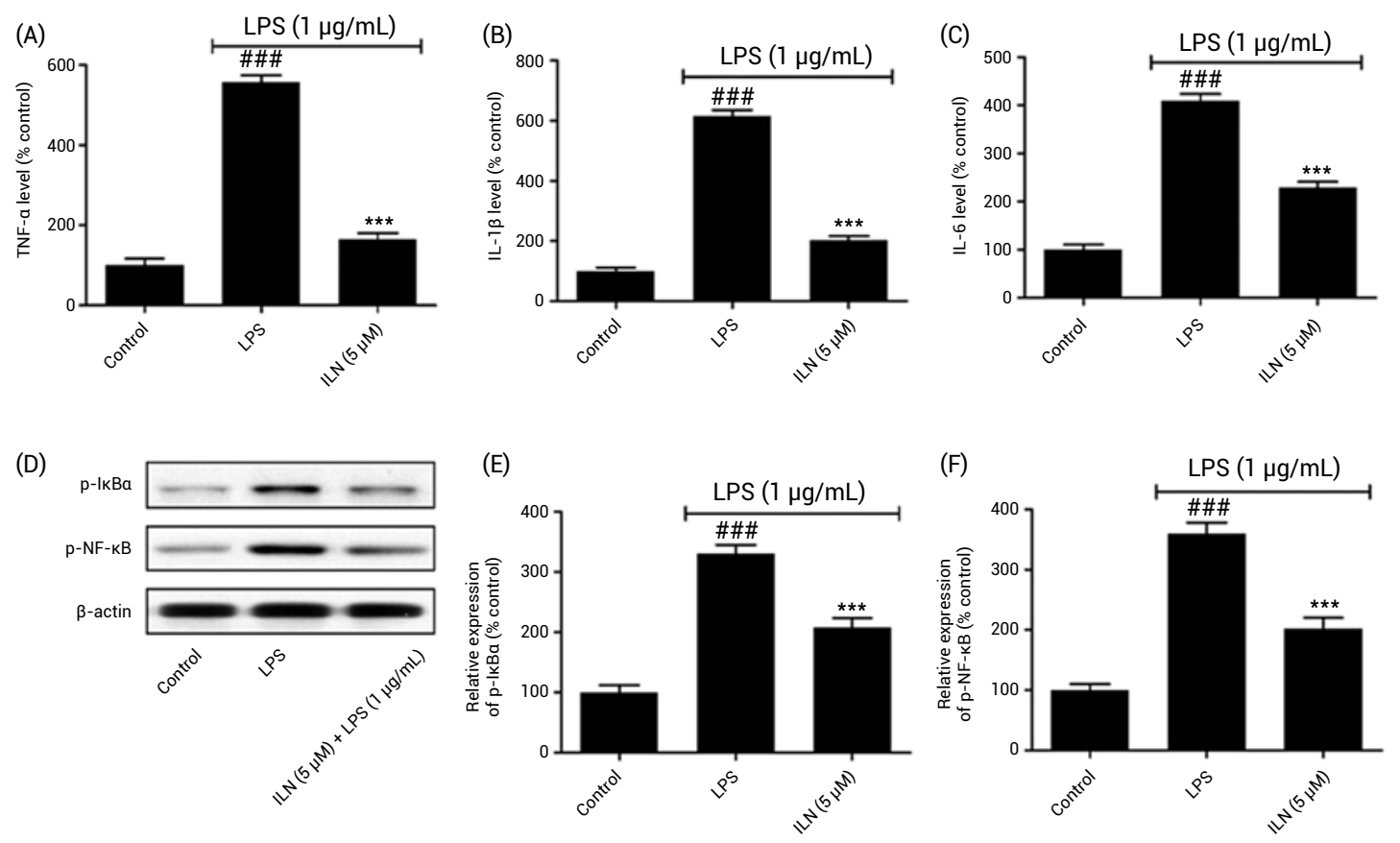

Figure 4. Effects of isoliquiritin (ILN) on the inflammation induced by LPS in BEAS-2B cells. (A) TNF- $\alpha$ level. (B) IL-1 $\beta$ level.

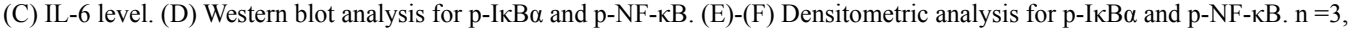
$\# \# \# \mathrm{P}<0.001$ vs control group, $* * * \mathrm{P}<0.001$ vs LPS group.

the production of MDA was elevated in the presence of LPS $(266.3 \pm 17.1 \%)$, which was remarkably blocked by isoliquiritin (160.5 $\pm 15.8 \%$ ) (Figure $3 \mathrm{~B})$. Additionally, LPS also resulted in the decreasing activity of antioxidant enzymes such as SOD and CAT $(55.4 \pm 7.9 \%$ and $64.1 \pm 6.5 \%)$. However, after being exposed to indicated isoliquiritin, the activity for SOD and CAT was enhanced as $86.9 \pm 7.9 \%$ and $83.5 \pm 8.2 \%$, respectively (Figure $3 \mathrm{C}$ and D). These results demonstrated isoliquiritin could attenuate oxidative stress in BEAS-2B cells stimulated by LPS.

\section{Effects of isoliquiritin on the inflammation induced by LPS in BEAS-2B cells}

To detect the inflammation in BEAS-2B cells, TNF- $\alpha$ level was explored herein. As a result, LPS can promote the secretion of TNF- $\alpha$ in BEAS-2B cells $(556.6 \pm 17.7 \%)$, which was reversed by isoliquiritin $(164.6 \pm 15.6 \%)$ (Figure 4A). Meanwhile, the levels of IL-1 $\beta$ and IL- 6 were elevated by LPS $(616.4 \pm 19.6 \%$ and $409.4 \pm 14.0 \%$ ), but the increasing levels were attenuated by isoliquiritin as $202.5 \pm 14.1 \%$ and $229.1 \pm 11.7 \%$, respectively (Figures 4B and C). As the transcription factor regulating the synthesis of TNF- $\alpha$, IL-1 $\beta$, and IL- 6 , the expression of NF- $\kappa$ B and its upstream $\mathrm{I} \kappa \mathrm{B} \alpha$ was detected using western blot analysis. The results showed isoliquiritin could ameliorate phosphorylation of both NF- $\kappa \mathrm{B}$ and $\mathrm{I} \kappa \mathrm{B} \alpha$ resulting from LPS stimulation (Figure 4D), which implied isoliquiritin was involved in the regulation of NF- $\kappa \mathrm{B}$ signaling in BEAS-2B cells. Accordingly, the densitometric analysis further confirmed results from the western blot analysis quantitatively (Figures 4E and $\mathrm{F}$ ).

\section{Isoliquiritin activates Nrf 2 in LPS-induced BEAS-2B cells}

To further disclose the protective mechanisms of isoliquiritin, activation of Nrf2 in BEAS-2B cells was explored. Fluorescence staining has shown in the presence of isoliquiritin, Nrf2 translocates into the nucleus from the cytosol (Figure 5A), which is similar to sulforaphane. To further localize Nrf2, western blot analysis was implemented. As shown in Figure 5B, isoliquiritin has resulted in up-regulation of nuclear and total Nrf2. And densitometric analysis also supports the observed results (Figures 5C and D). These results obtained demonstrate isoliquiritin can activate Nrf2 in BEAS-2B cells induced by LPS.

\section{DISCUSSION}

In the clinic, COPD is a typical pulmonary disease closely related to inflammation, which is usually characterized as airflow limitation and impairment of lung function (16). Oxidative stress and inflammatory 
(A)
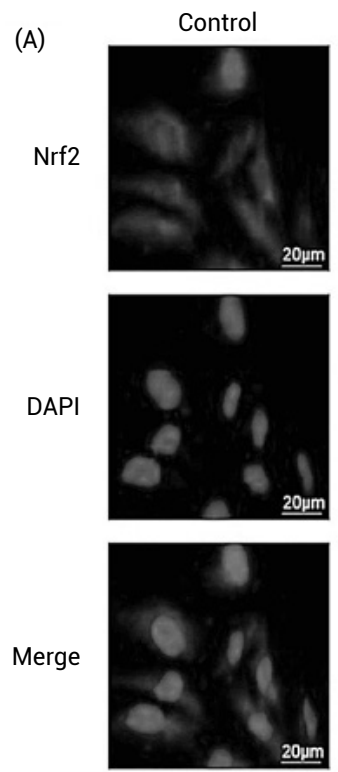
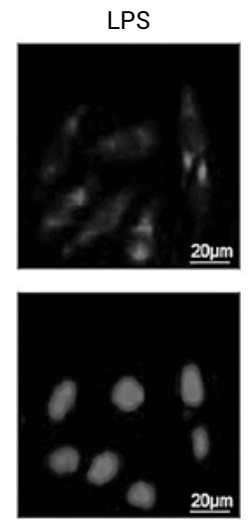

$\operatorname{ILN}(5 \mu \mathrm{M})$
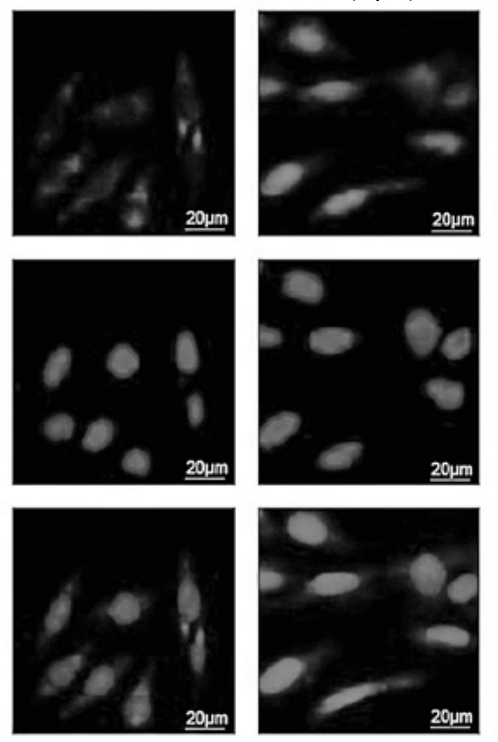
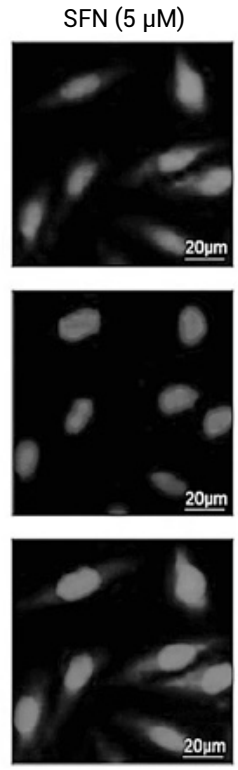

(B)

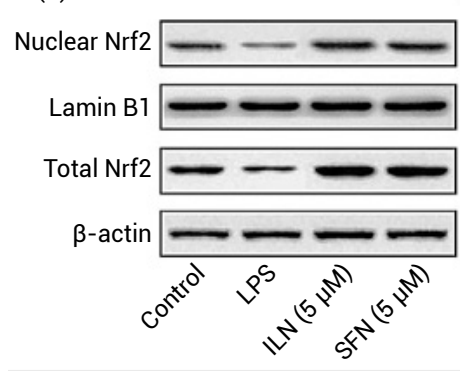

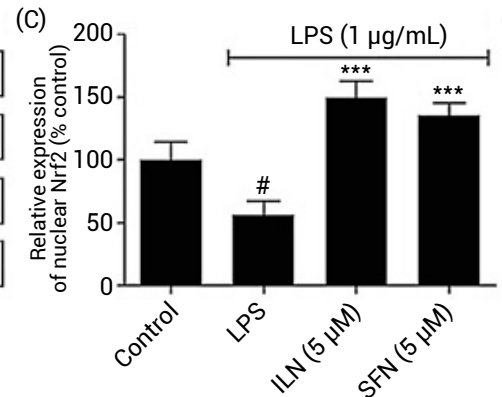

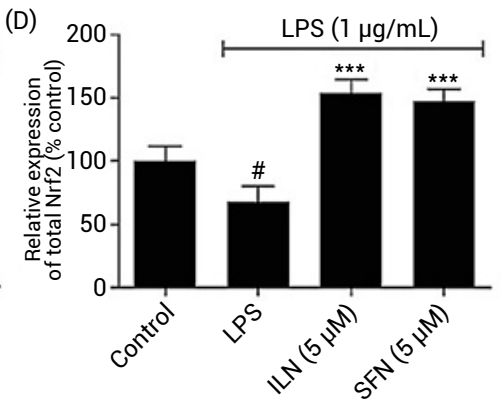

Figure 5. Isoliquiritin (ILN) activates Nrf2 in BEAS-2B cells induced by LPS. (A) Immunofluorescence staining. (B) Western blot analysis for nuclear and total Nrf2. (C)-(D) Densitometric analysis for nuclear and total $\mathrm{Nrf2}$. $\mathrm{n}=3, \# \mathrm{P}<0.05$ vs control group, $* * * \mathrm{P}<0.001$ vs LPS group.

response are the major underlying mechanisms of these diseases including COPD and ARDS since excessive mediators such as ROS, TNF- $\alpha$, and so on were generated following exposure to deleterious particles and gases $(17,18)$. Improvement of redox and inflammatory status will give an approach for the prevention of these progressive pulmonary diseases (19).

As the initiator of oxidative stress, ROS is excessive due to the imbalance between production and consumption (20). Overproduction of ROS can give rise to cell injury through the oxidation of proteins and DNA as well as lipid peroxidation (21). However, SOD and CAT are pivotal antioxidant enzymes that can lead to the conversion of superoxide into the water via hydrogen peroxide (22). Herein we have observed isoliquiritin decreased LPS-induced overproduction of ROS in BEAS-2B cells. As the major product of lipid peroxidation, the increasing MDA was also reduced by isoliquiritin.
Meanwhile, the activity of SOD and CAT was inhibited by isoliquiritin.

Inflammation is involved in the pathogenesis and progression of some pulmonary diseases like COPD as the consistent characteristics (23). NF- $\kappa \mathrm{B}$ can regulate the secretion of some pro-inflammatory cytokines such as TNF- $\alpha$, IL- $1 \beta$, and IL- 6 as the transcription factor (24). The activation of NF- $\kappa B$ is associated with the phosphorylation of I $\mathrm{B} \alpha$. In stimulated cells, $\mathrm{I} \kappa \mathrm{B} \alpha$ is activated through phosphorylation by phosphorylated IKK $\alpha$ and IKK $\beta$, which results in the degradation of $\mathrm{I} \kappa \mathrm{B} \alpha$ and phosphorylation of NF$\kappa \mathrm{B}$ dimer bound to $\mathrm{I} \kappa \mathrm{B} \alpha$. Then the phosphorylated $\mathrm{NF}-\kappa \mathrm{B}$ will translocate into the nucleus and regulate the transcription of genes (25). In addition, oxidative stress also contributes to the inflammatory process including activation of NF- $\kappa \mathrm{B}$ (26). Meanwhile, inflammation also promotes the overproduction of ROS as a radical burst (27). In our investigation, it was found isoliquiritin blocked LPS-induced activation 
of $\mathrm{NF}-\kappa \mathrm{B}$ signaling pathway and excessive secretion of pro-inflammatory mediators in BEAS-2B cells, which unraveled the anti-inflammatory potential of isoliquiritin.

$\mathrm{Nrf2}$ is a transcription factor regulating the transcription of target genes encoding antioxidant enzymes including SOD and CAT via binding to the promoter region of related genes (28). Under the unstressed condition, Nrf2 is sequestered by Keap1 in the cytoplasm and degraded through ubiquitination. In the presence of oxidants or electrophiles, the complex of Keap1-Nrf2 will be disrupted and free Nrf2 will translocate into the nucleus from the cytoplasm to promote transcription of genes (29). In addition, the crosstalk between Nrf2 and NF- $\kappa B$ indicates activation of Nrf2 will attenuate inflammation through inactivation of NF$\kappa \mathrm{B}$ and down-regulation of pro-inflammatory cytokines (30). In the present exploration, Nrf2 was activated in LPS-stimulated BEAS-2B cells in the presence of isoliquiritin, which implied activation of Nrf2 was involved in the protective effects of isoliquiritin.

As the pathophysiological features of COPD, chronic inflammation and acute exacerbations are crucial in the progress of COPD (31). A lower concentration of LPS can result in inflammation in BEAS-2B cells and higher will even lead to apoptosis of BEAS-2B cells $(32,33)$. Herein we used BEAS-2B cells treated by low concentration of LPS to explore the anti-inflammatory effects of isoliquiritin and possible mechanisms in vitro. The results showed the anti-inflammatory and anti-oxidant effects of isoliquiritin via Nrf2 activation, which may give evidence for the anti-inflammation in COPD.

\section{CONCLUSION}

In conclusion, we have evaluated the protective effects of isoliquiritin against oxidative stress and inflammation using LPS-induced BEAS-2B cells and explored the underlying mechanisms. The results uncovered isoliquiritin could attenuate oxidative stress and inflammation triggered by LPS, which was closely associated with activation of Nrf2. These results can provide indications for the application of isoliquiritin and the discovery of novel drugs targeting inflammation in pulmonary diseases.

\section{Conflict of interest}

The authors declare no conflicts of interest.

\section{REFERENCES}

1. Aghasafari P., George U., Pidaparti R.: Inflamm. Res. 68, 59 (2019).

2. Rabe K.F., Watz H.: Lancet 389, 1931 (2017).

3. Caramori G., Kirkham P., Barczyk A., Di Stefano A., Adcock I.: Ann. N. Y. Acad. Sci. 1340, 55 (2015).

4. Imai Y., Kuba K., Neely G.G., YaghubianMalhami R., Perkmann T., et al.: Cell 133, 235 (2008).

5. Bryan H.K., Olayanju A., Goldring C.E., Park B.K.: Biochem. Pharmacol. 85, 705 (2013).

6. Chen X.L., Kunsch C.: Curr. Pharm. Des. 10, 879 (2004).

7. Yuan J., Liu R., Ma Y., Zhang Z., Xie Z.: Inflammation 41, 1804 (2018).

8. Wang Z., Fang K., Wang G., Guan X., Pang Z., et al.: Phytother. Res. 33, 808 (2019).

9. Maleki S.J., Crespo J.F., Cabanillas B.: Food Chem. 299, 125124 (2019).

10. Fu B., Li H., Wang X., Lee F.S., Cui S.: J. Agric. Food. Chem. 53, 7408 (2005).

11. Kim J.Y., Park S.J., Yun K.J., Cho Y.W., Park H.J., Lee K.T.: Eur. J. Pharmacol. 584, 175 (2008).

12. Zhou Y.Z., Li X., Gong W.X., Tian J.S., Gao X.X., et al.: Food Funct. 8, 1235 (2017).

13. Luo J., Li Z., Wang J., Weng Q., Chen S., Hu M.: Molecules 21, 237 (2016).

14. Li Y., Song W., Tong Y., Zhang X., Zhao J., et al.: J. Neuroinflammation 18, 1 (2021).

15. Liu Y., Xu X., Xu R., Zhang S.: Drug Des. Devel. Ther. 13, 3735 (2019).

16. Celli B.R., Wedzicha J.A.: N. Engl. J. Med. 381, 1257 (2019).

17. Austin V., Crack P.J, Bozinovski S., Miller A.A., Vlahos R.: Clin. Sci. 130, 1039 (2016).

18. Matthay M.A., Ware L.B., Zimmerman G.A.: J. Clin. Invest. 122, 2731 (2012).

19. Victoni T., Barreto E., Lagente V., Carvalho V.F.: Oxid. Med. Cell. Longev. 2021, 6646923 (2021).

20. Sies H., Berndt C., Jones D.P.: Annu. Rev. Biochem. 86, 715 (2017).

21. Zorov D.B., Juhaszova M., Sollott S.J.: Physiol. Rev. 94, 909 (2014).

22. Dickinson B.C., Chang C.J.: Nat. Chem. Biol. 7, 504 (2011).

23. Brightling C., Greening N.: Eur. Respir. J. 54, 1900651 (2019).

24. Hayden M.S., Ghosh S.: Cell Res. 21, 223 (2011).

25. Yi P.F., Wu Y.C., Dong H.B., Guo Y., Wei Q., et al.: Immunopharmacol. Immunotoxicol. 35, 567 (2013). 
26. Lugrin J., Rosenblatt-Velin N., Parapanov R., Liaudet L.: Biol. Chem. 395, 203 (2014).

27. Mittal M., Siddiqui M.R., Tran K., Reddy S.P., Malik A.B.: Antioxid. Redox Signal. 20, 1126 (2014).

28. Shaw P., Chattopadhyay A.: J. Cell. Physiol. 235, 3119 (2020).

29. Yamamoto M., Kensler T.W., Motohashi H.: Physiol. Rev. 98, 1169 (2018).
30. Ahmed S.M., Luo L., Namani A., Wang X.J., Tang X.: Biochim. Biophys. Acta 1863, 585 (2017).

31. Farkas L., Stoelcker B., Jentsch N., Heitzer S., Pfeifer M., Schulz C.: Scand. J. Immunol. 68, 315 (2008).

32. Zhang P., Mak J.C., Man R.Y., Leung S.W.: Eur. J. Pharmacol. 865, 172731 (2019).

33. Lv X., Zhou X., Yan J., Jiang J., Jiang H.: Biomed. Pharmacother. 87, 180 (2017). 